\title{
Bandwidth Management Mechanisms for Ethernet Passive Optical Networks with Multi-ONU Customers
}

\author{
Oscar J. Ciceri, Nelson L. S. da Fonseca (Advisor) \\ ${ }^{1}$ Institute of Computing - State University of Campinas (UNICAMP) \\ Avenida Albert Einstein, 1251 - 13.083-852 - Campinas - SP - Brazil \\ oscarelrc.ic.unicamp.br, nfonseca@ic.unicamp.br
}

\begin{abstract}
Passive optical network (PON) is a cost-efficient access network technology to deliver broadband services. Moreover, service providers employ PONs to offer novel services. New business scenarios are, thus, envisioned in which customers owning multiple optical network units (ONUs) are connected to a single PON (multi-ONU customers). This paper proposes a dynamic bandwidth allocation (DBA) algorithm to guaranteed bandwidth for multi-ONU customers in Ethernet PONs (EPONs). It also introduces a bandwidth sharing algorithm to support cooperation among customers. Results show that the proposed algorithm can improve the overall throughput and quality of service provisioning.
\end{abstract}

Resumo. A rede óptica passiva (PON) é uma tecnologia econômica para fornecer serviços de banda larga. Além disso, os provedores de serviços empregam PONs para oferecer novos serviços. Assim, são previstos novos cenários de negócios, nos quais os clientes locatários de múltiplas unidades ópticas de redes (ONUs) estão conectados a uma única PON (clientes multi-ONU). Este artigo propõe um algoritmo de alocação dinâmica de largura de banda (DBA) para garantir os requisitos de largura de banda de clientes multi-ONU nas redes Ethernet PON (EPON). O artigo, também introduz um algoritmo de compartilhamento de largura de banda para fornecer cooperação entre os clientes. Os resultados mostram que os algoritmos propostos podem melhorar a vazão e a qualidade do serviço.

\section{Introduction}

In the past few years, passive optical networks (PONs) have been deployed in broadband access networks with an acceptable benefit-cost ratio. Moreover, in order to further maximize revenues, service providers employing PONs can offer new services such as mobile backhauling/fronthauling [Astudillo and da Fonseca 2014] and PON virtualization [Wang et al. 2017].

PON customers can be residential subscribers, single or multi-site enterprises, other service providers such as mobile network operators (MNOs), virtual network operators (VNOs), and tenants owning a PON slice. Thus, we refer as PON customer not only the end user, but also service providers which may rent resources of the PON infrastructure from the Infrastructure service provider (InP).

Infrastructure service providers have been searching for new services and business models to increase their revenues by capitalizing on their deployed infrastructure. Moreover, new scenarios are envisioned in which customers owning multiple ONUs are 
connected to a single PON (multi-ONU customers). In line with that, the motivation for the thesis is to allow new business models for EPONs which use efficiently the bandwidth of access networks. However, a drawback is that InPs are currently able to support guaranteed bandwidth only to individual ONUs with the existing dynamic bandwidth allocation (DBA) algorithms. Consequently, peaks of bandwidth demand may surpass the guaranteed bandwidth for some ONUs and, at the same time, guaranteed bandwidth being underutilized by other ONUs. Wastage of bandwidth is a major problem in asses networks, which is the current bottleneck of the Internet.

This article presents the work in the dissertation Bandwidth Management Mechanisms for Ethernet Passive Optical Networks with Multi-ONU Customers [Ciceri 2019], which objetive is to address the bandwidth management problem for multi-ONU customers in EPON networks. It introduces the following original contributions:

- A novel DBA algorithm EPON networks. This algorithm supports multi-ONU service level agreements (SLAs) for multi-ONU customers, as well as, individual $S L A s$ for traditional customers with a single ONU;

- A new class of scheduling mechanisms for EPON networks, which allows isolation at different levels of granularity: individual ONUs, subgroup of ONUs and customer. The proposed DBA algorithm assures bandwidth to traditional customers, multi-ONU customers with a single service and multi-ONU customers serving diverse type of services. The DBA algorithm also allows multi-ONU customers to support a prioritized bandwidth to subgroups of ONUs;

- A DBA algorithm for allowing cooperation among EPON customers. This algorithm allows cooperative customers to share unused bandwidth among themselves without affecting their guaranteed bandwidth.

These contributions advance the state of the art in the design of DBA algorithms for EPON networks. The relevance of the original contributions in this paper is, therefore, the evolution of optical access networks technology, which is fundamental to reduce costs to the end-users and increase revenue to infrastructure providers.

Currently, broadband access networks need to support ever increasing demands for bandwidth due to the large-scale adoption of novel services and, the increased number of Internet users. We postulate that the impact of the contributions to InPs is the ability to cope with huge bandwidth demands to support different services and applications with diverse requirements. The solutions proposed in [Ciceri 2019] increase the overall network utilization and improve quality of service (QoS) provisioning since they allow a fair and efficient distribution of bandwidth among customers.

\section{Related Work}

This section briefly reviews the literature related to dynamic bandwidth allocation (DBA) algorithms in EPON networks. The most popular DBA algorithm for EPONs is the interleaved polling with adaptive cycle time (IPACT) algorithm [Kramer et al. 2002], which defines an online grant scheduling framework. The majority of existing algorithms proposed so far are variation of IPACT. To achieve multiplexing gain, IPACT grants transmission opportunities to each ONU using a round robin mechanism. IPACT defines four grant windows-size policies called fixed, limited, closed, and excess, being the most widely used DBA. With the limited policy, the bandwidth is guaranteed to each ONU according to pre-defined SLAs. 
Other DBA algorithms based on interleaved polling (IP) with fixed scheduling frame size have been proposed in the EPON literature (e.g., [Merayo et al. 2007] and [i. Choi and Park 2010]), for facilitating the implementation of Differentiated Services supporting diverse SLAs for ONUs. However, existing DBA algorithms are currently able to support only guaranteed bandwidth to individual ONUs. Moreover, DBA algorithms that includes bandwidth sharing provisioning, customers isolation and customization within customer have been recently proposed [Alvarez et al. 2014], [Li et al. 2016], [Afraz et al. 2018].

No EPON DBA scheme to supports multi-ONU customers and bandwidth sharing among customers in EPON networks has been proposed so far. Even though an XGPON DBA algorithm [Alvarez et al. 2014] supports a similar concept (group assured bandwidth), it cannot be employed in EPONs due to the fundamental differences between these two technologies [Butt et al. 2017].

\section{Proposed DBA Algorithm for Bandwidth Guaranteed for Multi-ONU Customers in EPON Networks}

The dissertation [Ciceri 2019] proposed a novel EPON DBA algorithm which supports multi-ONU SLAs for multi-ONU customers called IPACT with multi-ONU SLAs support (MOS-IPACT). We call multi-ONU SLA a DBA which considers the aggregated SLAs of a group of ONUs as a single SLA.

Currently, EPON DBA algorithms do not allow customers with more than one ONU in a PON to take advantage of the statistical multiplexing among ONUs. Traditionally, each ONU has an individual SLA specifying its guaranteed bandwidth. Conversely, in MOS-IPACT, a single SLA, called multi-ONU SLA, can be defined for a whole group of ONUs that belong to the same customer. This multi-ONU SLA defines a guaranteed bandwidth per ONU, which can be aggregated with the guaranteed bandwidth of the other ONUs in the group. This aggregated bandwidth is shared among all ONUs in the same group in a granting cycle basis. In this way, the unused bandwidth from underloaded ONUs can be redistributed among overloaded ONUs belonging to the same group by using an excess bandwidth distribution policy, which increases the network utilization. Section 3.2 in [Ciceri 2019] gives a detailed description of the MOS-IPACT algorithm and its excess policy, which allows a dynamic bandwidth distribution among ONUs of the same customer.

We used the EPON-Sim simulator to evaluate the performance of the MOS-IPACT DBA algorithm. The EPON-Sim simulator was developed in Java and previously validated in [Dias and da Fonseca 2012] and [Astudillo and da Fonseca 2014]. EPON-Sim implements the IPACT DBA algorithm together with the limited discipline introduced by Kramer et. al in [Kramer et al. 2002]. Moreover, the MOS-IPACT algorithm with the fair excess (FE) DBA policy was introduced in the EPON-Sim simulator and the new version of the simulator was validated extensively. The IPACT algorithm is used in this comparison since it is the most widely studied DBA algorithm in the literature. This algorithm is employed in most comparisons which the arbitration mechanism is based on the MPCP protocol developed by the IEEE 802.3ah Task Force.

The performance of the proposed MOS-IPACT algorithm was compared to that IPACT. The simulation included a 10G-EPON network with 1 optical line terminator (OLT) serving a set of 32 ONUs $(|\mathcal{O}|=32$ ) on an optical distribution network in a 


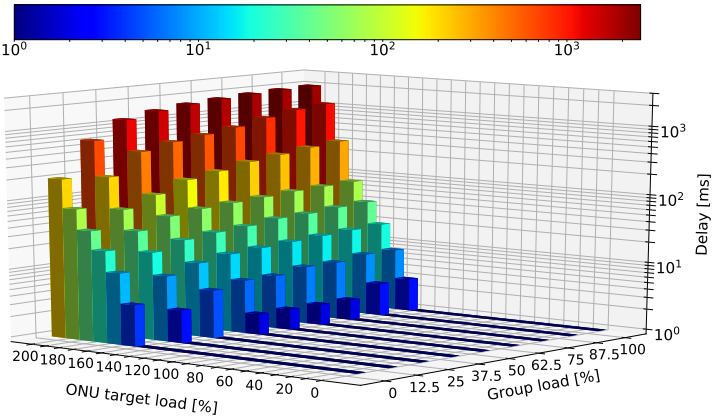

(a) $N_{\text {group }}=2$

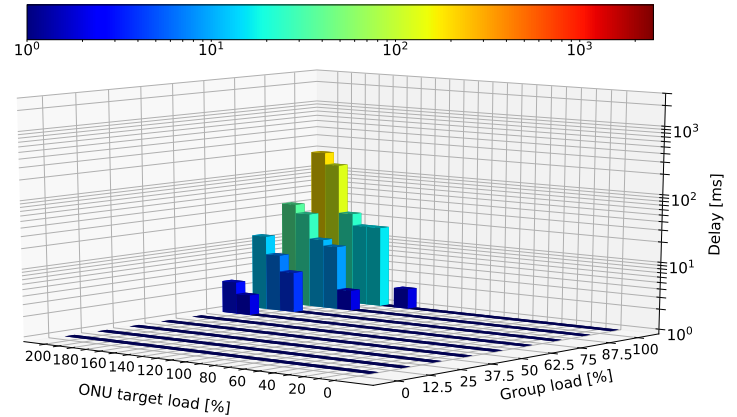

(b) $N_{\text {group }}=8$

Figure 1. Impact of the number of ONUs in the group on the DELAY of the AF traffic for the IPACT DBA algorithm.

tree topology. Each ONU had three different traffic classes: expedited forwarding (EF), assured forwarding (AF), and best effort (BE). The EF traffic represented voice and other delay-sensitive applications that require low end-to-end delay. It was modelled by using a constant bit rate encoding with a fixed-size packet of 70 bytes. The rest of the offered load was evenly distributed among AF and BE traffic, which typically host applications requiring bounded delay, as well as, bandwidth guarantees. The traffic AF or BE were self-similar generated by using aggregations of ON-OFF sources.

Moreover, it was assumed that there was one customer with multi-ONU SLA $S$ assigned to the group of customer's ONUs $\left(N_{\text {group }}\right)$, which varied from 2 to 8 . Among these ONUs, there was a target ONU (ONU target $)$ with $300 \mathrm{Mbps}$ of guaranteed bandwidth. The other $N_{\text {group }}-1$ ONUs belonging to $S$ have guaranteed bandwidth between $150 \mathrm{Mbps}$ and $450 \mathrm{Mbps}$, provided that $\left(N_{\text {group }}-1\right) \times 300 \mathrm{Mbps}$, which was the effective aggregated guaranteed bandwidth of the ONU group excluding the target ONU. On the other hand, there was a set of conventional ONUs $\mathcal{O}_{C}$. Each ONU belonging to $\mathcal{O}_{C}$ had a guaranteed bandwidth and offered load equal to $300 \mathrm{Mbps}$. Section 3.3.1 in [Ciceri 2019] gives a complete description of the simulation parameters, traffic models and assumptions employed in the simulations.

Simulation results show that the EF traffic experienced delay values less than 1 ms and no packet loss (figures not show in this paper) since the guaranteed bandwidth was sufficient to serve the high priority traffic. Similar to the EF traffic, the AF traffic did not suffer packets loss, due to its high priority over the BE traffic. Thus, in this section, we focus on the analysis of the average packet delay of AF traffic (delay-sensitive) of the target ONU when the load and the number of ONUs in $\mathcal{O}_{S}$ varies.

The average packet delay of the target ONU AF traffic for the IPACT and MOSIPACT algorithms are shown in Figure 1 and 2, respectively. A reduction in the delay of the AF traffic is observed when the number of ONUs in the group increases since the extra available bandwidth also increases, allowing the ONUs to transmit a higher number of packets in shorter periods.

Moreover, under loads of $87.5 \%$, when the MOS-IPACT protocol was used, the average packet delay of eight $\mathrm{ONU}$ in the group is negligible. In the worst case, when the target ONU is under loads of $200 \%$ and the offered group load is $100 \%$, the average packet delay is $5 \mathrm{~ms}$ when the MOS-IPACT scheme is employed, whereas it is $200 \mathrm{~ms}$ 


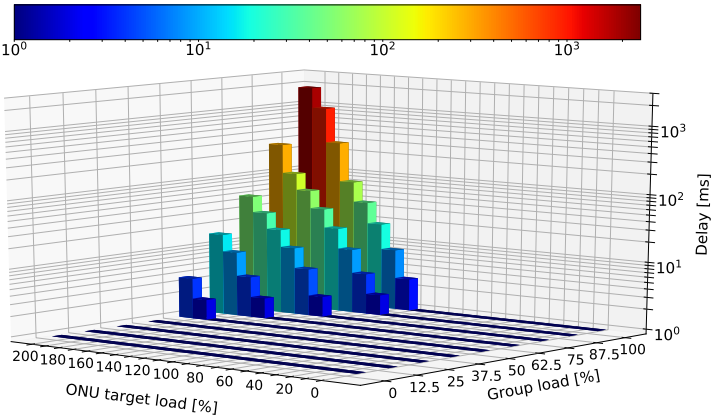

(a) $N_{\text {group }}=2$

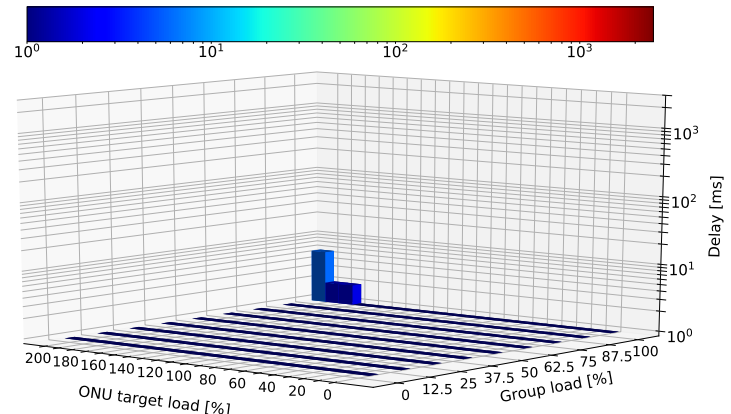

(b) $N_{\text {group }}=8$

Figure 2. Impact of the number of ONUs in the group on the DELAY of the AF traffic for the MOS-IPACT DBA algorithm.

when IPACT is used. This means that the average packet delay values given by MOS$I P A C T$ for eight ONUs in the group were up to two order of magnitude lower than those given by IPACT.

The MOS-IPACT algorithm produced lower delay values in the ONU target for load lower than $100 \%$ since the excess bandwidth of underloaded ONUs were redistributed to the overloaded ONUs of a multi-ONU customer in a per cycle basis. Subsection 3.3.2 and 3.4.3 in [Ciceri 2019] present additional results.

\section{Proposed DBA Algorithm for Cooperative Resource Sharing among EPON Customers}

Existing algorithms in the literature do not take into account that EPON customers produce a large spatio-temporal traffic variability, which may lead to underutilization of network resources, as well as degradation of the QoS provisioning. Resource sharing is a promising approach to cope with such variability [Schneir and Xiong 2014]. Customers can share the bandwidth among themselves to increase the overall network utilization [Li et al. 2016] [Ciceri et al. 2018] or even receive economic incentives [Afraz and Ruffini 2018]. Hence, resource sharing can make the PON infrastructure more profitable and attractive to customers by employing new business models.

The dissertation [Ciceri 2019] introduces a DBA algorithm called IPACT with cooperative customers support (CS-IPACT) that allows bandwidth sharing in EPONs among cooperative customers. Customers can join a cooperative group for sharing their unused bandwidth among other customers in the group. In this way, unused bandwidth from underloaded customers is redistributed to overloaded customers belonging to the same cooperative group in a granting cycle basis, which can increase the network utilization.

Moreover, traditional customers with a single ONU and customers with multiple ONUs can cooperate to increase the available bandwidth without compromising their guaranteed bandwidth. The CS-IPACT algorithm guarantees the bandwidth agreed in the SLAs. Section 5.2 in [Ciceri 2019] gives a detailed description of the CS-IPACT algorithm.

The CS-IPACT algorithm was introduced in the EPON-Sim simulator and the new version of the simulator was validated extensively. We compared the performance of the $M O S-I P A C T$ to that of the proposed algorithm (CS-IPACT). The MOS-IPACT algorithm 


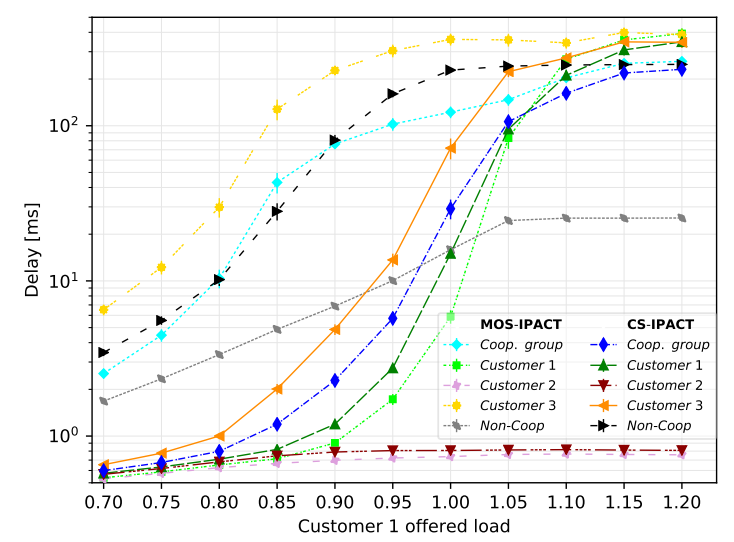

(a) Average delay

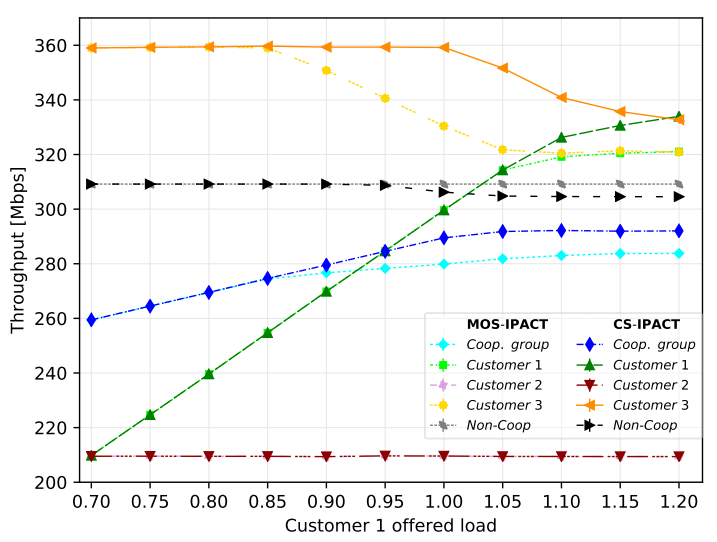

(b) Average throughput per ONU

Figure 3. Impact of cooperation among EPON customers

was employed since it is the only protocol in the literature that supports guaranteed bandwidth for multi-ONU customers.

In the simulated scenario, an EPON network with tree topology and optical channel capacity of $10 \mathrm{~GB} / \mathrm{s}$ was employed. There was one cooperative group with three cooperative customers, representing, for instance, MNOs with backhauling/fronthauling services, and virtual network operators with residential and enterprise ONUs. The offered load of one cooperative customer varied from an underloaded to an overloaded condition, while two cooperative customer had fixed loads: one overloaded and the other underloaded. Customer 1 has an aggregated offered load varying from $0.7 \cdot B_{C_{1}}$ to $1.2 \cdot B_{C_{1}}{ }^{1}$, where $B_{C_{i}}$ was the effective aggregated guaranteed bandwidth of customer $C_{i}$, whereas Customer 2 and Customer 3 had an aggregated offered load of 0.7 (underloaded) and 1.2 (overloaded), respectively. On the other hand, there was a set of non-cooperative ONUs $\left(\mathcal{O}_{\mathcal{N}}\right)$. These ONUs had guaranteed bandwidth $B_{k}$ equal to the remaining effective bandwidth in the PON divided by the number of ONUs in $\mathcal{O}_{\mathcal{N}}$. To properly assess the performance of the proposed algorithm, the offered load of a non-cooperative ONUs was set to its guaranteed bandwidth value, which was an overloaded condition for that set of ONUs. We analyzed the average throughput per ONU and the average delay for each cooperative customer, the cooperative group, and the non-cooperative customers for both algorithms. Section 5.3.1 in [Ciceri 2019] gives a complete description of the simulation parameters, traffic models and assumptions employed in the simulations.

Results show that the average packet delay values produced by CS-IPACT for the cooperative group are up to one order of magnitude lower than those given by MOSIPACT (Figure 3a). Moreover, the CS-IPACT algorithm produced up to $5 \%$ higher average throughput values per ONU belonging to the cooperative group than did the MOS-IPACT algorithm (Figure $3 \mathrm{~b}$ ). This is a consequence of the distribution of the excess bandwidth of cooperative customers in the same cooperative group. Conversely, any unused bandwidth in a PON cycle cannot be explicitly redistributed to other customers when MOS-IPACT is employed because existing EPON DBA algorithms do not allow cooperation among customers.

\footnotetext{
${ }^{1}$ For the sake of clearness and brevity, herein after, $B_{C_{i}}$ is omitted from the offered load values of Customer $C_{i}$
} 
Moreover, for the overloaded customer (Customer 3), when the offered load is lower than 1.0, CS-IPACT produced delay values much lower than those given by MOS$I P A C T$. The MOS-IPACT produces slightly lower delay values for the other two cooperative customers than did CS-IPACT due to the higher throughput provided by the latter (Figure 3b). Under overloaded conditions (offered load higher than 1.05), the delay values produced by the two DBA algorithms were in the same order of magnitude. On the other hand, the average throughput value per ONU produced by CS-IPACT can be $5 \%$ to $10 \%$ higher than those produced by MOS-IPACT.

The above-described results show the impact of sharing resources among customers in a cooperative way on the delay and throughput. CS-IPACT allows the overloaded ONUs to use the excess bandwidth of the underloaded ONUs in the same cooperative group in a per cycle basis. All these gains are obtained without affecting the throughput (Figure 3b) and delay (Figure 3a) of the underloaded cooperative customers. This means that ONUs of a cooperative customer support higher bit rates than those guaranteed in the SLA even under high and unbalanced traffic loads without affecting other cooperative customers.

Moreover, Customer 3 throughput decreases as the load of Customer 1 increases when CS-IPACT is used for overloaded condition because our mechanism employs the fair excess policy to distribute the excess bandwidth between overloaded customers. Note that when Customer 1 and Customer 3 have the same offered load value (offered load equal to 1.2), the CS-IPACT produces the same throughput for these cooperative customers. This shows that a fair distribution of the excess bandwidth is provided to the cooperative customers by the proposed distribution policy.

\section{Publications}

Results in the dissertation [Ciceri 2019] were reported in three papers.

- Ciceri, O. J., Astudillo, C.A., Fonseca, N.L.S. Dynamic Bandwidth Allocation with Multi-ONU Customer Support for Ethernet Passive Optical Networks. IEEE Symposium on Computers and Communications (ISCC), May 2018, pp. 1-6. This paper received the conference Best Paper Award.

- Ciceri, O. J., Astudillo, C.A., Fonseca, N.L.S. DBA Algorithm with Prioritized Services for 10G-EPON with Multi-ONU Customers. IEEE Latin-American Conference on Communications (LATINCOM), Nov 2019, pp. 1-6.

- Ciceri, O. J., Astudillo, C.A., Fonseca, N.L.S. DBA Algorithm for Cooperative Resource Sharing among EPON Customers. IEEE International Conference on Communications (ICC), June 2020, pp. 1-6. This paper received the Transmission, Access, and Optical Systems (TAOS) Technical Committee's Award for Best Paper in the SAC-ANS\&PLC Symposium.

In addition, we are preparing an article extending the results of the MOS-IPACT algorithm, that will be submitted to an international journal.

\section{Conclusion}

This article summarized the contribution of the master thesis [Ciceri 2019], which studied the bandwidth allocation problem in EPON networks with multi-ONU customers. We introduced three novel DBA algorithm for bandwidth allocation in these networks. The 
support of multi-ONU customer, as well as the resource sharing approach between cooperative customers are original contributions of this work and they can lead to an increase in the utilization of network resources, while guaranteeing QoS requirements.

\section{References}

Afraz, N., Elrasad, A., and Ruffini, M. (2018). DBA capacity auctions to enhance resource sharing across virtual network operators in multi-tenant PONs. In 2018 Optical Fiber Communications Conference and Exposition (OFC), pages 1-3.

Afraz, N. and Ruffini, M. (2018). A sharing platform for multi-tenant PONs. Journal of Lightwave Technology, 36(23):5413-5423.

Alvarez, P., Marchetti, N., Payne, D., and Ruffini, M. (2014). Backhauling mobile systems with XG-PON using grouped assured bandwidth. In Networks and Optical Communications - (NOC), 2014 19th European Conference on, pages 91-96.

Astudillo, C. A. and da Fonseca, N. L. S. (2014). Standard-compliant QoS provisioning scheme for LTE/EPON integrated networks. IEEE Wireless Commun., 21(3):44-51.

Butt, R. A., Ashraf, M. W., Faheem, M., and Idrus, S. M. (2017). A survey of dynamic bandwidth assignment schemes for TDM-based passive optical network. Journal of Optical Communications.

Ciceri, O. J., Astudillo, C. A., and da Fonseca, N. L. S. (2018). Dynamic bandwidth allocation with multi-ONU customer support for ethernet passive optical networks. In EEE Symposium on Computers and Communications (ISCC), pages 1-6.

Dias, M. P. and da Fonseca, N. L. S. (2012). A robust WiMAX scheduler for EPONWiMAX networks. In 2012 IEEE Global Communications Conference (GLOBECOM), pages 1580-1585.

i. Choi, S. and Park, J. (2010). SLA interleaved polling with adaptive cycle time-aware dynamic bandwidth allocation for QoS in EPONs. IEEE/OSA Journal of Optical Communications and Networking, 2(9):773-781.

Kramer, G., Mukherjee, B., and Pesavento, G. (2002). IPACT a dynamic protocol for an ethernet PON (EPON). IEEE Commun. Mag., 40(2):74-80.

Li, C., Guo, W., Wang, W., Hu, W., and Xia, M. (2016). Bandwidth resource sharing on the XG-PON transmission convergence layer in a multi-operator scenario. J. Opt. Commun. Netw., 8(11):835-843.

Merayo, N., Duran, R. J., Fernandez, P., d. Miguel, I., Aguado, J. C., Lorenzo, R. M., and Abril, E. J. (2007). Interleaved polling algorithm with service level agreement (SLA) to improve QoS in Ethernet PONs. In 2007 9th International Conference on Transparent Optical Networks, volume 4, pages 28-31.

Schneir, J. R. and Xiong, Y. (2014). Cost analysis of network sharing in FTTH/PONs. IEEE Communications Magazine, 52(8):126-134.

Wang, X., Cavdar, C., Wang, L., Tornatore, M., Chung, H. S., Lee, H. H., Park, S. M., and Mukherjee, B. (2017). Virtualized cloud radio access network for 5G transport. IEEE Commun. Mag., 55(9):202-209. 